\title{
The Shortest Way is the One You Know Best: Is It Also Valid for Natural Products Research?
}

\author{
Alev Onder ${ }^{1,3 *}$, Lutfun Nahar ${ }^{2,3}$ and Satyajit D Sarker ${ }^{3}$ \\ ${ }^{1}$ Department of Pharmacognosy, Faculty of Pharmacy, Ankara University, Turkey \\ ${ }^{2}$ Laboratory of Growth Regulators, Institute of Experimental Botany ASCR and Palacký University, Czech Republic \\ ${ }^{3}$ Centre for Natural Products Discovery, School of Pharmacy and Biomolecular Sciences, Liverpool John Moores University, United Kingdom \\ *Corresponding Author: Alev Onder, Professor, Department of Pharmacognosy, Faculty of Pharmacy, Ankara University, Turkey.
}

Received: August 28, 2019; Published: October 01, 2019

DOI: $10.31080 /$ ASPS.2019.03.0410

Natural products play important roles as biomaterials, because of their ability to interact with biological targets [1]. The power of natural products to cure diseases has encouraged systematic screening of natural products aiming at discovering and developing new medicines [2,3]. Therefore, new approaches have been developed for the discovery of new molecules originating from natural products [4-6]. However, difficulties in investigations of natural products have somewhat reduced the interest in these compounds in the pharmaceutical industrial sectors [1]. Natural products-based drug discovery is often a highly complex process that requires interdisciplinary collaborations [5,7]. In addition, ethical and economic hardles are faced in the development of natural drugs [8]. In spite of all difficulties, scientists have been constantly working to find new ways to make it easier to analyse natural products, because of unique chemical diversity, and uncharted chemical space, which are ideal for drug discovery and development [1]. In the earliest times, the relationships between people and the environment has started with the struggle to cure diseases, and for this purpose, especially they have used plants. Thus, for many years, medicinal plants have been exploited as a mean to heal various human ailments [2,9]. To date, many modern drugs, e.g., morphine, quinine, taxol and vincristine, are of plant origins $[10,11]$.

The first step in discovering new drug molecules from medicinal plants is ethno-pharmacological inquiries, which gives us information about what was previously used [5,8]. In this way, the next steps are phytochemical studies and pharmacological tests, which can prove or disprove available information through a wider interdisciplinary effort. If a valuable molecule is discovered, in later stages, the plant that is the source of this molecule should be produced by biotechnological methods or the compound of interest and its analogues can be produced by chemical synthesis.

Bio-assay-guided isolation may be the most effective method in identifying active compounds whether or not ethnobotanical information is available, because every step is assessed by bioassays leading to identification of bioactive compounds, which are characterized by a combination of modern spectroscopic techniques $[12,13]$.

Plant selection for any screening and the choice of appropriate extraction protocol often affect the bioactivity and the overall outcome. The identification of the correct species is a crucial step; otherwise, all the experiments will be useless. An extract prepared from the same plant material can exhibit different chemical properties according to different preparation methods. In addition, plant extracts prepared using solvents of different polarities are important in terms of providing chemical diversity based on polarity. Therefore, it is necessary to pay attention to these two important stages. Because of self-denying efforts over the years, many compounds are isolated from medicinal plants, and their effects are clarified, with their mechanisms and some of them are converted to patents. With the advances in technology and science, drug discoveries from plants will continue to provide new drug molecules in the years to come.

Of course, the most talked about objective is to achieve a faster and more accurate result in the isolation of secondary compounds. What is described above is in the most general sense, about how to get to the active ingredients. The book published by Sarker 
and Nahar [3], all these methods are well explained in the most detailed form. Moreover, it is not difficult to find new methods in different literature. However, one thing remains unchanged that some classical or basic methods for obtaining active components are not abandoned even still valid today.

Although the drug discovery and development from natural products or mainly from plants is a tedious process and possess many challenges, it is clear that natural products, especially plants, will still play a vital role in the discovery of new drugs.

\section{Bibliography}

1. Carlson EE. "Natural products as chemical probes". ACS Chemical Biology 16.5 (2010): 639-53.

2. Wang MW., et al. "Biological screening of natural products and drug innovation in China". Philosophical Transactions of the Royal Society B 362.1482 (2007): 1093-105.

3. Sarker SD and Nahar L. "Natural Product Isolation. Methods and Protocols". Third Edition. Humana Press. London (2012).

4. Abel U., et al. "Modern methods to produce natural-product libraries". Current Opinion in Chemical Biology 6 (2002): 453458.

5. Atanasov AG., et al. "Discovery and resupply of pharmacologically active plant-derived natural products: A review". Biotechnology Advances 33.8 (2015): 1582-1614.

6. Thomford NE., et al. "Natural Products for Drug Discovery in the 21st Century: Innovations for Novel Drug Discovery". International Journal of Molecular Sciences 25.19 (2018). pii: E1578.

7. Heinrich M. "Ethnopharmacology in the 21st century-grand challenges”. Frontier Pharmacology 1 (2010): 8.

8. Ngo LT., et al. "21st century natural product research and drug development and traditional medicines". Natural Product Reports 30.4 (2013): 584-592.

9. Phillipson JD. "Phytochemistry and medicinal plants". Phytochemistry 56 (2001): 237-243.

10. Kinghorn AD., et al. "The relevance of higher plants in lead compound discovery programs". Journal of Natural Products 74 (2011): 1539-1555.
11. Newman DJ and Cragg GM. "Natural products as sources of new drugs over the 30 years from 1981 to 2010". Journal of Natural Products 75 (2010): 311-335.

12. Sharma SB and Gupta R. "Drug Development from Natural Resource: A Systematic Approach”. Mini-Reviews in Medicinal Chemistry 15 (2015): 52-57.

13. Altemimi A., et al. "Phytochemicals: Extraction, Isolation, and Identification of Bioactive Compounds from Plant Extracts". Plants (Basel). 6.42 (2017): 1-23.

\section{Volume 3 Issue 11 November 2019 (C) All rights are reserved by Alev Onder., et al.}

